\title{
White Hole Cosmology - One Best Alternative for Standard Cosmology
}

\author{
U.V.S. Seshavatharam ${ }^{1}$ and S. Lakshminarayana ${ }^{2}$ \\ ${ }^{1}$ Honorary faculty, I-SERVE, Survey no-42, Hitech city, Hyderabad-84,Telangana, India \\ ${ }^{2}$ Dept. of Nuclear Physics, Andhra University, Visakhapatnam-03, AP, India \\ Emails: seshavatharam.uvs@gmail.com (and)1nsrirama@gmail.com
}

\begin{abstract}
By modifying the basic definition of cosmic red shift as $[\mathrm{z} /(1+\mathrm{z})]$, considering 'speed of light' as an absolute cosmic expansion rate, adopting 'Planck mass' as the basic seed of the observed large scale universe and following a scaled form of Hawking's black hole temperature formula, it is certainly possible to review and revise the basic picture of 'standard cosmology' and in near future, a perfect model of 'white hole cosmology' can be developed. In this context we have developed five assumptions. Independent of matter density fractions, first assumption is helpful in understanding galactic red shifts and galactic light travel distances. Second and third assumptions are based on 'time reversed' black holes and seem to be well connected with General theory of relativity as well as Quantum mechanics. Fourth and fifth assumptions are helpful in understanding current galactic dark matter and flat rotation speeds. It may be noted that, considering our first three assumptions and considering the Planck Legacy 2018 data's enhanced lensing amplitude in cosmic microwave background power spectra - conceptually, a closed universe having a positive curvature seems to be a best fit for the observed universe. With reference to our recent publication [26], for clarity on the subject, in this paper, we make an attempt to review and explain our proposed assumptions at fundamental level. Our aim is to see that, professional and non-professional cosmologists must understand the basics of workable quantum cosmology.
\end{abstract}

Keywords: new definition of cosmic red shift, Planck mass, quantum model of cosmology, light speed expansion, galactic dark matter, flat rotation speed, cosmic rotation, galactic acceleration.

\begin{tabular}{|c|l|}
\hline \multicolumn{2}{|l|}{ Plan of the paper } \\
\hline 1 & Introduction and purpose \\
\hline 2 & Five assumptions \\
\hline 3 & A general review on assumptions 4 and 5 \\
\hline 4 & A critical review on assumption-1 \\
\hline 5 & A critical review on assumption-2 \\
\hline 6 & A critical review on assumption-3 \\
\hline 7 & Understanding the true nature of cosmic geometry \\
\hline 8 & Understanding cosmological galactic acceleration \\
\hline 9 & A review on standard model of Lambda cosmology \\
\hline 10 & Future applications of the proposed five assumptions \\
\hline 11 & Conclusion \\
\hline
\end{tabular}

\section{Introduction and purpose}

Modern cosmologists are seriously working on developing a peculiar model of relativistic cosmology that assumed to be started with a 'big bang' [1] followed by a sudden and very short period exponential expansion called 'inflation' [2] and a lately begun current 
acceleration [3,4]. Big bang and inflation, both, seem to be the best widely accepted theoretical inferences of current large scale cosmic observations. But it is very clear to say that, both are having no proper theoretical base with reference to the basic concepts of general theory of relativity $[5,6,7]$. In addition to that, currently believed cosmic acceleration is based on another widely accepted invisible and unidentified cosmic 'new energy source' called 'dark energy'[8]. Apart from these three complicated cosmic issues, galactic observations strongly supporting the existence of yet another mysterious and invisible cosmic 'mass source' called 'dark matter' [9]. It is very unfortunate to say that, so far, no one did establish a clear physical connection between cosmic dark energy and dark matter.

We emphasize the point that, standard model of cosmology is basically a model of classical model of general relativity and it seems inevitable to bring a change with reference to quantum model of cosmology $[10,11,12]$. Another important point to be noted is that, quantum cosmology point of view, 'Spin' is a basic criteria and 'cosmic rotation' is no way against to general theory of relativity. Since standard model is being developed and maintained by high level mathematics, so called 'standard model' of cosmology is strongly believed and shown to be in line with large scale cosmic observations that are completely depending on an ad-hoc and misleading definition of 'cosmic red shift' [13,14] associated with light coming from distant galaxies.

Here we would like to appeal the point that, by modifying the basic definition of cosmic red shift, considering 'speed of light' $[15,16,17]$ as an absolute cosmic expansion rate and adopting 'Planck mass' as the basic seed of the observed large scale universe, it is certainly possible to review and revise the basic picture of 'standard cosmology' and in near future, a perfect model of 'white hole cosmology' can be developed [18-26] with a positive curvature and rotation. In this context we have developed five assumptions. It is very interesting to note that, the recent Planck Legacy 2018 release has confirmed the presence of an enhanced lensing amplitude in cosmic microwave background (CMB) power spectra compared to that predicted in the standard Lambda cosmology model. Experts suggest that, this kind of enhanced lensing amplitude can be understood with a closed universe having a positive curvature at more than $99 \%$ confidence level [27-30].

With reference to our recent publication [26], for clarity on the subject, in this short communication, we make an attempt to review and explain our proposed assumptions at fundamental level. Our aim is to see that, professional and non-professional cosmologists must understand the basics of workable quantum cosmology.

\section{Five assumptions}

Out of five, first three assumptions are helpful in understanding the basic cosmic structure and $4^{\text {th }} \& 5^{\text {th }}$ assumptions are helpful in understanding current galactic structures. With further study, they can be modified for a better understanding.

Assumption-1: Actual cosmic red shift seems to be, $z_{\text {new }} \cong z_{n} \cong \frac{\lambda_{O}-\lambda_{L}}{\lambda_{O}} \cong 1-\left(\frac{\lambda_{L}}{\lambda_{O}}\right) \cong\left(\frac{z}{z+1}\right) \leq 1 \quad$ where $\lambda_{O}$ is the wave length of light received from observed galaxy, $\lambda_{L}$ is the wave length of light in laboratory and $z$ is the currently believed cosmic red shift.

Assumption-2: With reference to Planck mass, cosmic temperature [31] follows a scaled form of Hawking's black hole temperature relation, $T_{t} \cong \frac{\hbar c^{3}}{8 \pi G k_{B} \sqrt{M_{t} M_{p l}}}$ where $M_{p l} \cong$ Planck mass and $M_{t} \cong$ Cosmic mass. 
Assumption-3: If $R_{t} \cong 2 G M_{t} / c^{2} \cong c / H_{t} \cong c t, M_{t} \cong c^{3} / 2 G H_{t}$ and $t H_{t} \cong 1$.

Assumption-4: Current galactic dark matter $\left(M_{G d}\right)_{0}$ and current visible matter $\left(M_{G v}\right)_{0}$ are interrelated [32-37] in such a way that, $\left(M_{G}\right)_{0} \cong\left(M_{G d}\right)_{0}+\left(M_{G v}\right)_{0}$ and $\frac{\left(M_{G d}\right)_{0}}{\left(M_{G v}\right)_{0}} \cong \sqrt{\frac{\left(M_{G v}\right)_{0}}{M_{X 0}}}$ where $M_{X 0} \cong \sqrt{\frac{3 H_{0}^{2} c^{2}}{8 \pi G a T_{0}^{4}}} \frac{\left(M_{0}^{3} M_{p l}\right)^{\frac{1}{4}}}{8 \pi}=3.63 \times 10^{38} \mathrm{~kg}=$ Current dark-visible reference mass unit.

Assumption-5: Current galactic flat rotation speed [38-40] can be expressed as, $\frac{\left(V_{G}\right)_{0}}{c} \cong \frac{1}{4}\left(\frac{\left(M_{G}\right)_{0}}{M_{0}}\right)^{\frac{1}{4}}$ and $\left(V_{G}\right)_{0} \cong\left[G\left(\left(M_{G}\right)_{0} / 128\right) c H_{0}\right]^{\frac{1}{4}}$ where $M_{0}=$ Current cosmic total mass.

Note: It may be noted that, based on assumptions (1) and (2), at any stage of cosmic evolution,

1) Currently believed 'critical energy density' and cosmic mass-energy density are equal in magnitude.

2) Ratio of mass energy density to thermal energy density is always a constant, $\frac{3 H_{t}^{2} c^{2}}{8 \pi G a T_{t}^{4}} \cong 5760 \pi$

3) Hubble parameter and temperature are always strongly interrelated via scaled Hawking's black hole temperature formula.

4) Cosmological constant problem can be understood with, $\left(\frac{3 H_{p l}^{2} c^{2}}{8 \pi G}\right) /\left(\frac{3 H_{t}^{2} c^{2}}{8 \pi G}\right) \cong \frac{H_{p l}^{2}}{H_{t}^{2}} \cong \frac{T_{p l}^{4}}{T_{t}^{4}}$.

\section{A general review on assumptions 4 and 5}

Assumptions 4 and 5 need a critical review for their scope and applicability for the past and future cosmic evolution. Using assumption-4 and by knowing the galactic visible mass, galactic dark matter can be quantified. Using assumption-5 and by knowing the galactic total mass, galactic flat rotation speeds can be fitted. Alternatively, considering both the assumptions and by knowing the galactic flat rotation speed and visible mass, galactic total mass and dark mass, both can be estimated. Comparing assumption 5 with MOND's relation $\left(V_{G}\right)_{0} \cong\left[G\left(M_{G v}\right)_{0}\left(\frac{c H_{0}}{6}\right)\right]^{\frac{1}{4}}$, it is possible to show that, $\frac{\left(M_{G}\right)_{0}}{128} \cong \frac{\left(M_{G v}\right)_{0}}{6}$. On writing $\left(M_{G}\right)_{0} \cong\left(M_{G d}\right)_{0}+\left(M_{G v}\right)_{0}, \quad$ one $\quad$ can $\quad$ obtain, $\quad \frac{\left(M_{G d}\right)_{0}}{\left(M_{G v}\right)_{0}} \cong \frac{128}{6}-1 \cong 20.33 \quad$ and 
$\frac{\left(M_{G v}\right)_{0}}{\left(M_{G d}\right)_{0}} \cong \frac{1}{20.33} \cong 0.049$. This ratio is nicely matching with the currently believed cosmic baryonic matter density fraction. But, based on assumption 4 , relation between galactic dark mass and visible mass is not linear and magnitude of galactic dark mass is proportional to $\left[\left(M_{G v}\right)_{0}\right]^{\frac{3}{2}}$. Based on this idea,

1) Observed low dark matter content of NGC1052-DF2 and NGC1052-DF4 can be understood $[33,34]$.

2) Very recent studies conducted on super spiral galaxies with Southern African Large Telescope (SALT) suggest [20,21,22,41 high rotation speeds in the range of (240 to $570) \mathrm{km} / \mathrm{sec}$ indicating a dynamical mass range of $(0.6$ to 4$) \times 10^{12} \mathrm{M}_{\odot} \mathrm{kg}$. It may be noted that, with a visible galactic mass of $\sqrt{0.6 \times 4} \times 10^{12} \mathrm{M}_{\odot} \mathrm{kg} \cong 3.08 \times 10^{42} \mathrm{~kg}$, estimated flat rotation speed is $558 \mathrm{~km} / \mathrm{sec}$.

3) Sun's estimated dark mass is $1.48 \times 10^{26} \mathrm{~kg}$.

4) Nucleon's estimated dark mass is $3.6 \times 10^{-60} \mathrm{~kg}$.

5) Electron's estimated dark mass is $4.6 \times 10^{-65} \mathrm{~kg}$.

Considering the estimated dark masses of Sun and nucleons, it is reasonable to say that, starting from nuclear volume to solar volume, dark matter effects are negligible. It can be confirmed with current observations. Interesting point to be noted is that, for understanding the nature of dark matter at atomic and nuclear scales, experimental setup should have an access to find ultra light masses of the order of $\left(10^{-65}\right.$ to $\left.10^{-60}\right) \mathrm{kg}$. Readers are encouraged to visit https://www.quantamagazine.org/physicists-are-expanding-the-search-for-dark-matter20201123/.

\section{A critical review on assumption-1}

In reality, so far, experimentally, no one did confirm any elementary particle travelling with superluminal speed. Observationally, so far no one did confirm any galaxy receding with superluminal speed. Quantum gravity point of view and as photons and gravitons are confirmed to move with speed of light, it seems illogical to believe in superluminal speeds. Based on these points, currently believed cosmic red shift definition can be considered as 'erroneous' and it seems necessary to review its mathematical representation. Considering 'light speed' as a characteristic upper limit of galactic receding speed, we have developed assumption-1. As per assumption-1, without considering standard model of matter density fractions, light travel distances, $\left(z_{\text {new }}\right)\left(\frac{c}{H_{0}}\right)$ can be reproduced with marginal error. See figure-1. Based on figure-1, it is certainly possible to relinquish the matter density fractions and dark energy concepts associated with standard model of cosmology. With further study, confusion associated with basic cosmic red shift can be eliminated. Keeping its simplicity in view, our new definition of cosmic red shift can be recommended for further research [26]. For a comparison readers are encouraged to visit https://cosmocalc.icrar.org/ and http://www.atlasoftheuniverse.com/cosmodis.c. 
Figure1: Comparison of standard and estimated light travel distances

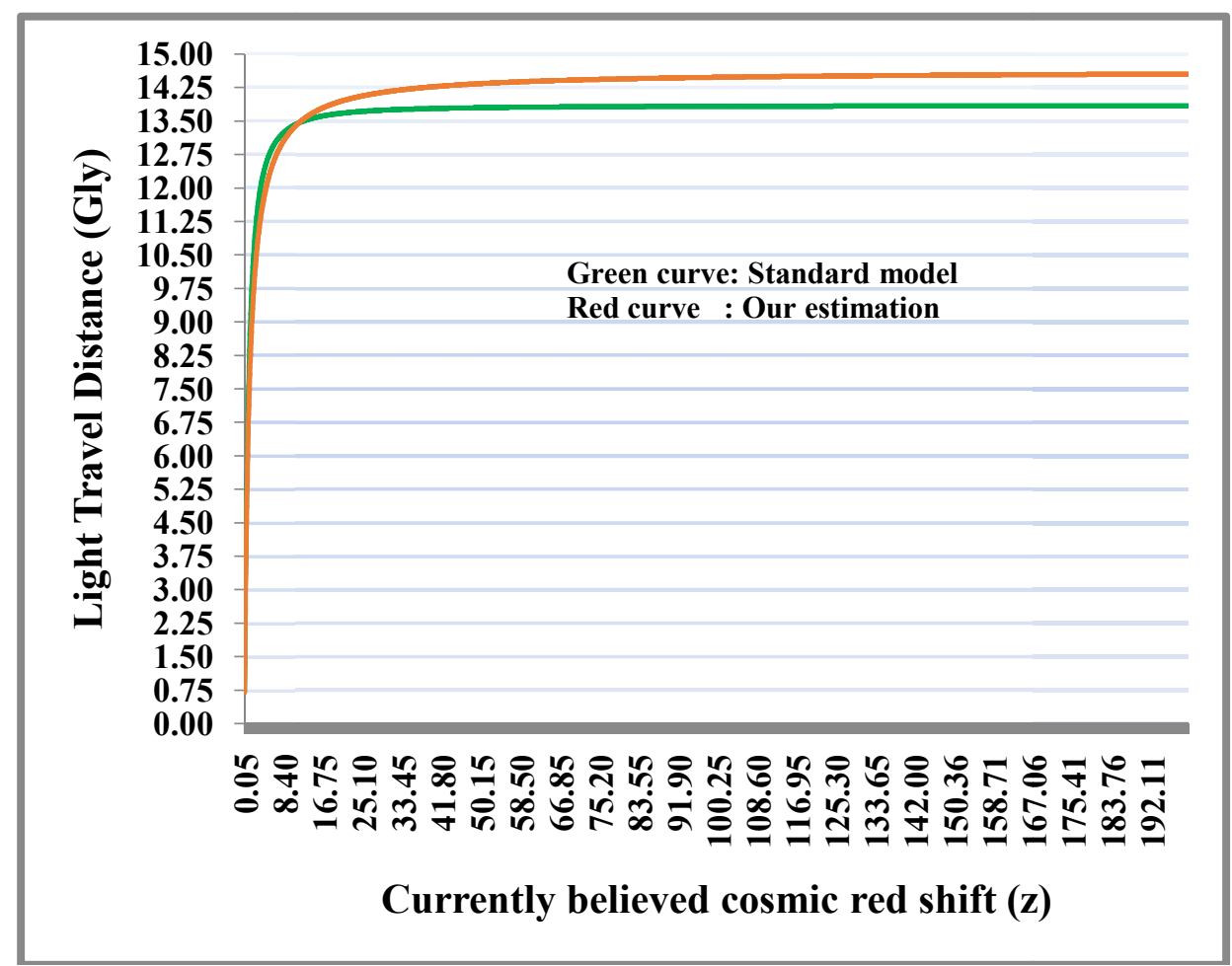

\section{A critical review on assumption-2}

Currently believed standard model of cosmology is based on general theory of relativity. To have a quantum model of cosmology, it seems essential to review the basics of Lambda cosmology in view of quantum background. Cosmologists should accept this point. Considering the product of Lambda cosmology model's current cosmic critical density and current Hubble volume, current total cosmic mass can be expressed as, $M_{0} \cong\left(\frac{3 H_{o}^{2}}{8 \pi G}\right)\left(\frac{4 \pi}{3}\left(\frac{c}{H_{0}}\right)^{3}\right) \cong\left(\frac{c^{3}}{2 G H_{0}}\right)$. Quantum gravity point of view, it is well confirmed that, black holes are the best quantum objects. As black holes are confirmed to be the central objects of all grown and growing galaxies, it seems possible to consider the expanding universe as a time reversed black hole (a white hole). In this context, it is possible to express its current cosmic radius as, $R_{t} \cong \frac{2 G M_{0}}{c^{2}} \cong \frac{c}{H_{0}}$.

Proceeding further, by substituting the geometric mean of 'Planck mass' and $\left(\frac{c^{3}}{2 G H_{0}}\right)$ in the famous Hawking's black hole temperature formula [12,26], it is possible to fit the current cosmic temperature accurately [31,42]. If $H_{0} \cong 67 \mathrm{~km} \cdot \mathrm{sec}^{-1} \cdot \mathrm{Mpc}^{-1}$, estimated $T_{0} \cong 2.728^{\circ} \mathrm{K}$. If $H_{0} \cong 75 \mathrm{~km} \cdot \mathrm{sec}^{-1} \cdot \mathrm{Mpc}^{-1}$, estimated $T_{0} \cong 2.886^{\circ} \mathrm{K}$. This can be considered as one of the best understandable effect of quantum gravity on very large scale distances of the observable universe. In addition to that, this idea completely eliminates the tension in estimating the current Hubble parameter. 


\section{A critical review on assumption-3}

The fundamental question to be answered is - What is the correct form of cosmic curvature? With reference to critical density, cosmologists try to say that,

1) If observable matter density is greater than the critical density, there is a possibility for a positive curvature.

2) If observable matter density is less than the critical density, there is a possibility for a negative curvature.

3) If observable matter density is equal to the critical density, there is a possibility for no curvature (i.e. it is flat - means infinite in volume without a center).

Considering, the sum of ordinary matter density, dark matter density and dark energy density, it is strongly believed that, universe is flat on large scale distances. But it is very unfortunate to say that, so far, no observation or experiment did confirm the existence of dark energy. Until the direct existence of dark energy is evidenced, it is not correct to believe in "cosmic flatness'. In addition to that, when it is strongly believed that universe is infinite in volume without a center (flat), it is illogical to confine our studies with 'Hubble volume'. In this complicated and ambiguous scenario, we are working on assumptions 2 and 3 on their scope, applicability and validity in understanding cosmic center and curvature with reference to cosmic white hole of volume, $\left(\frac{4 \pi}{3}\left(\frac{c}{H_{0}}\right)^{3}\right)$, mass, $\left(\frac{c^{3}}{2 G H_{0}}\right)$ and mass density, $\left(\frac{3 H_{0}^{2}}{8 \pi G}\right)$. We would like appeal that, when 'total mass density' is identical with currently believed 'critical density', classification scheme associated with 'critical density' can be ignored/relinquished.

Basically, assumption-3 seems to be a representation of a simple model of universe having a center and positive curvature of radius, $R_{t} \cong 2 G M_{t} / c^{2}$. In order to fit the current cosmic temperature, we are trying to apply it for estimating the current cosmic expansion rate with a simple relation of the form, $R_{t} \cong 2 G M_{t} / c^{2} \cong c / H_{t}$. In this context, by considering Melia's model of light speed expanding universe (equation-8 of [15], Monthly Notices of Royal Astronomical Society), asuumption-3, can be validated. But, this procedure seems to be ambiguous with reference to currently believed flat model of the universe. As assumptions 2 and 3 are helpful in estimating the current Hubble parameter independent of galactic distances and brightness, we would like to emphasize the following three points.

1) Cosmic temperature plays a vital role in understanding the cosmic curvature.

2) Cosmic white hole is having a positive curvature of radius $R_{t} \cong 2 G M_{t} / c^{2} \cong c / H_{t}$.

3) Horizon of the cosmic white hole is expanding with speed of light.

Based on the very basic physics, frankly speaking, for the universe having a center and positive curvature, it seems reasonable and correct to insert a relation of the form, $V_{t} / \omega_{t}$ in place of $c / H_{t}$ where $V_{t}$ and $\omega_{t}$ represent cosmic rotational speed and angular velocity respectively. Clear speaking, logically, assumption-3, seems to be valid and more meaningful when, $R_{t} \cong 2 G M_{t} / c^{2} \cong V_{t} / \omega_{t}$. By replacing $V_{t}$ with speed of light (as cosmic expansion speed) and by replacing $\omega_{t}$ with cosmic expansion rate $H_{t}$, in this paper, we are making an attempt to estimate other cosmic physical parameters. In most of our published papers we have followed all possible ways. Whether it is correct or not, will be decided with advanced study and research [28]. Reference 28 clearly suggests that, thermal behavior of the universe 
plays a vital role in understanding the actual cosmic curvature. It seems to be in-line with our assumptions 2 and 3. As standard model of cosmology is not in line with quantum models of cosmology having a positive curvature, it may take some time go deep into the study of assumption-3. With further study, cosmic rotation [43-57] can be verified with a relation of the form, $R_{t} \cong 2 G M_{t} / c^{2} \cong c / H_{t} \cong\left(1 / \gamma_{t}\right) *\left(c / \omega_{t}\right), \gamma_{t} \omega_{t} \cong H_{t}$ and $V_{t} \cong c / \gamma_{t}$ where $\gamma_{t} \cong(1$ to 150$)$ is a coefficient of proportionality to be decided with any suitable model or observational data. In our very recent paper [26], we proposed that, $\frac{H_{t}}{\omega_{t}} \cong \Upsilon_{t} \cong 1+\ln \left(\frac{H_{p l}}{H_{t}}\right)$. Current value of estimated $\frac{H_{0}}{\omega_{0}} \cong \Upsilon_{0} \cong 140.61$. Hence estimated current angular velocity and rotational speed are $\omega_{0} \cong 1.5412 \times 10^{-20}$ rad.sec ${ }^{-1}$ and $V_{0} \cong 2.132 \times 10^{6} \mathrm{~m}_{\mathrm{sec}} \mathrm{sec}^{-1}$ respectively. If so, galactic flat rotation speeds can be expressed as, $\frac{\left(V_{G}\right)_{0}}{c} \cong\left(\frac{1}{2 \gamma_{0}}\right)^{\frac{1}{4}}\left(\frac{\left(M_{G}\right)_{0}}{M_{0}}\right)^{\frac{1}{4}} \cong 0.2442\left(\frac{\left(M_{G}\right)_{0}}{M_{0}}\right)^{\frac{1}{4}}$ and $\left(V_{G}\right)_{0} \cong\left[G\left(M_{G}\right)_{0} c \omega_{0}\right]^{\frac{1}{4}} \cong\left[G\left(\frac{\left(M_{G}\right)_{0}}{140.61}\right) c H_{0}\right]^{\frac{1}{4}}$. Thus, $\frac{\left(M_{G v}\right)_{0}}{\left(M_{G d}\right)_{0}} \cong\left(\frac{140.61}{6}-1\right)^{-1} \cong 0.0446$. Based on this coincidence and considering a broad range of very light and super massive galaxies, assumptions 4 and 5 can be modified with reference to MOND's relation [58], $\left(V_{G}\right)_{0} \cong\left[G\left(M_{G v}\right)_{0}\left(\frac{c H_{0}}{6}\right)\right]^{\frac{1}{4}} \cong\left[G\left(\frac{\left(M_{G v}\right)_{0}}{6}\right) c H_{0}\right]^{\frac{1}{4}}$ and actual galactic dark mass.

\section{Understanding the true nature of cosmic geometry}

As per general theory of relativity, when there is no outward force, space-time follows a positive curvature proportional to its matter content. According to standard cosmology, universe is infinite in volume having unlimited matter content. So far no did confirm the direct physical existence of the (inferred) hidden driving force or outward force of the infinite universe. In addition to that, when space is expanding at super luminal speeds in infinite volume, second to second, on large scale distances, cosmic temperature must show a continuous decrease in its magnitude. Observed CMBR isotropy is dead against to this logic. Based on these points, it seems reasonable to appeal that,

1) There is a chance of misleading and misinterpretation in models of cosmology having infinite volume and infinite matter.

2) Currently believed cosmic flatness is not $100 \%$ correct.

3) Dark energy may not be a compulsory entity in developing workable models of cosmology $[59,60]$.

4) Models of cosmology having a positive curvature cannot be ignored.

5) Rotation is a common phenomenon in closed models of cosmology.

6) Galactic observations and CMB observations must show an unique inference pertaining to cosmic geometry. 
7) Scope and validity of 'Light speed' can be thoroughly analyzed in all possible ways as a limiting condition of 'cosmic expansion speed', 'galactic receding speed' and 'cosmic rotation speed'.

In this context, it is very important to consider R. Burghardt's model [61,62] of 'Subluminal universe' having a positive curvature and light speed expansion. It resembles Melia's model $[15,63,64,65]$ of the universe in all respects and deviates from 'Flat model'. It is very unfortunate to say that, these models are lacking in estimating and understanding the origin of cosmic thermal bath in a quantum gravity approach. Here we would like to emphasize the point that, by combining our model with Burghardt's model, Melia's model, Tatum's model $[66,67]$ and Sapar's model [68], in near future, a very nice model of quantum cosmology can be developed in all respects.

\section{Understanding cosmological galactic acceleration}

According to Saul Perlmutter, Adam Riess and Brian Schmidt, observable universe is accelerating $[3,4]$. Clearly speaking, expansion of the universe is such that the velocity at which a distant galaxy is receding from the observer is continuously increasing with time. It can be understood in the following way $[20,22]$. It needs a thorough analysis with reference to currently observed galactic acceleration.

With reference to two time periods $\left(t_{2}>t_{1}\right)$, ratio of galactic receding speeds can be expressed as, $\frac{\left(v_{\text {Gres }}\right)_{t_{2}}}{\left(v_{\text {Gres }}\right)_{t_{1}}} \cong\left(\frac{\left(r_{\text {Gdis }}\right)_{t_{2}}}{\left(r_{\text {Gdis }}\right)_{t_{1}}}\right)\left(\frac{R_{t_{1}}}{R_{t_{2}}}\right) \Rightarrow \frac{\left(v_{\text {Gres }}\right)_{t_{2}} R_{t_{2}}}{\left(v_{\text {Gres }}\right)_{t_{1}} R_{t_{1}}} \cong \frac{\left(r_{\text {Gdis }}\right)_{t_{2}}}{\left(r_{\text {Gdis }}\right)_{t_{1}}}$.

where,

$\left(v_{\text {Gres }}\right)_{t_{1}}$ and $\left(v_{\text {Gres }}\right)_{t_{2}}=$ Galactic receding speeds corresponding to $t_{1}, t_{2}$ respectively and $\left(v_{\text {Gres }}\right)_{t_{2}}>\left(v_{\text {Gres }}\right)_{t_{1}}$.

$\left(r_{\text {Gdis }}\right)_{t_{1}}$ and $\left(r_{\text {Gdis }}\right)_{t_{2}}=$ Galactic distances corresponding to $t_{1}, t_{2}$ respectively

and $\left(r_{\text {Gdis }}\right)_{t_{2}}>\left(r_{\text {Gdis }}\right)_{t_{1}}$.

$R_{t_{1}}$ and $R_{t_{2}}=$ Theoretical cosmic radii corresponding to $t_{1}, t_{2}$ respectively where $\left(R_{t_{2}}>R_{t_{1}}\right)$.

Clearly speaking,

1) Within the cosmic horizon, second by second, galactic receding speeds are increasing and resemble a kind of internal cosmic acceleration.

2) Acceleration seems to be higher near to cosmic centre and gradually reaches to zero at horizon.

3) Hubble's law pertaining to two increasing time periods seems to be a natural consequence of internal cosmic acceleration.

4) Cosmic horizon is always expanding at speed of light.

\section{A review on standard model of Lambda cosmology}

Based on the assumptions and considering the following points, at fundamental level, it is possible to review the standard model of cosmology. We would like to appeal that, 
1) Even though highly intuitive and impressive, there is no clarity and proper physical support for 'big bang' and 'inflation'.

2) Total mass energy density and critical energy density, both are identical and there is no need to consider a curvature classification scheme based on critical energy density.

3) Frankly speaking, if universe is really expanding with 'speed of light', unphysical Lambda term and its inherited dark energy term, both can be relinquished forever.

4) If one is willing to consider $\left(\frac{\Lambda_{t} c^{4}}{8 \pi G}\right)$ as a characteristic expression for 'dark energy density', at any stage of cosmic evolution, $\frac{3 c^{2} H_{t}^{2}}{8 \pi G}-\frac{\Lambda_{t} c^{4}}{8 \pi G} \cong 0$, then it can be inferred that, at any stage of cosmic evolution, difference of dark energy density and total mass-energy density is always 'zero' and universe is always expanding at speed of light.

5) Lambda term can be expressed as a scalar quantity having the form, $\Lambda_{t} \cong \frac{3}{R_{t}^{2}} \cong \frac{3 H_{t}^{2}}{c^{2}}$.

6) Cosmological constant problem can be understood with, $\frac{\Lambda_{t}}{\Lambda_{p l}} \cong \frac{R_{p l}^{2}}{R_{t}^{2}} \cong \frac{H_{t}^{2}}{H_{p l}^{2}} \cong \frac{T_{t}^{4}}{T_{p l}^{2}}$.

7) In Lambda cosmology, matter creation is associated with big bang. Based on the assumptions, matter is being created continuously with an expression of the form, $M_{t} \cong \frac{c^{3}}{2 G H_{t}} \cong \frac{c^{3} t}{2 G}$.

8) Cosmic Temperature-Time relation can be expressed as, $T_{t} \cong \frac{0.18511 \times 10^{10}}{\sqrt{t}} \mathrm{~K}$. This relation is almost similar to the mainstream relation derived on big bang concepts. Only difference is that, for the same expected temperatures, our estimated cosmic physical processes are taking place early compared to big bang model. This idea helps in understanding the early formation of galaxies at the cosmic dawn.

9) Most important thing is that the characteristic cosmic expansion rate can be accurately estimated by knowing the cosmic microwave radiation temperature (CMBR) and there is no need to take the help of galactic red shifts and galactic distances.

10) Estimated galactic dark matter is more than 90 percent of the total mass of 'massive' galaxies. In case of least massive galaxies also, dark matter is roughly $50 \%$ of the total mass of galaxy.

11) One thing is very clear that, modern red shift data and its advanced statistical analysis associated with cosmic rate of expansion is generating lot of confusion and controversy in assessing the correct rate of cosmic expansion.

12) When it is assumed that, current universe is a white hole, there exists no scope for 'horizon' problem and 'causal disconnection'.

13) The fundamental questions to be answered are - How much distance universe will expand with speed of light? and How long universe will expand with speed of light? Based on 'Zero' Kelvin temperature, answer seems to be: Infinite distance and infinite time. As destiny is unknown and when it is assumed that, universe is expanding/growing with speed of light, - 'light speed expanding space' can be called as 'Flat space'. (Flatness means: Infinite in volume without center).

\section{Future applications of the proposed five assumptions}

Considering the proposed five assumptions, 
1) Galactic flat rotation speeds and their distances can be reviewed in a better way.

2) Complicated mathematical formula associated with light travel distances can be simplified.

3) Standard cosmology and basic models of quantum cosmology can be studied in a unified approach.

4) Big bang, inflation and dark energy like intellectual concepts can be explored at utmost fundamental level.

5) Complicated mathematics of general theory of relativity can be simplified to a great extent.

6) Galactic acceleration can be understood with clarity and thereby error in estimating Hubble parameter can be eliminated.

7) Planck scale imprints associated with current cosmic observations can be explored and thereby a correct model of cosmology can be developed with high precision.

8) Inter relation between galactic dark matter and visible matter can be refined and understood in a better way.

9) Nature of dark matter can be explored at utmost fundamental level.

10) Atomic and nuclear scale dark matter secrets can be understood and thereby experiments can be conducted in a result oriented approach.

\section{Conclusion}

As there exists no experimental support and as there exist no other applications of dark energy, in phase-1, by relinquishing the currently believed critical density curvature scheme and adopting white hole cosmology with light speed expansion, it seems possible to develop a workable model of quantum cosmology independent of cosmic matter density fractions and dark energy. Time to time, based on the cosmic temperature data, Hubble parameter can be estimated accurately. In phase-2, on confirming the positive curvature of the current universe, details of cosmic angular velocity can be understood and verified in all possible ways. In phase-3, on confirming the current cosmic angular velocity, collectively, all cosmic physical parameters including the nature of galactic dark mass and cosmic age can be studied in a unified manner.

\section{Acknowledgments}

On technical grounds, author Seshavatharam is very much thankful to Dr. Eugene Terry Tatum for his valuable suggestions and remarks. Seshavatharam is indebted to Shri M. Nagaphani Sarma, Chairman;, Shri K.V. Krishna Murthy, founder Chairman, Institute of Scientific Research in Vedas (I-SERVE), Hyderabad, India and Shri K.V.R.S. Murthy, former scientist IICT (CSIR), Govt. of India, Director, Research and Development, I-SERVE, for their valuable guidance and great support in developing this subject.

\section{References}

[1] Chernin, A. D. 1995, George Gamow and the Big Bang. Space Science Reviews, 74(3-4), 447-454

[2] Andrei Linde. 2008, Inflationary Cosmology. Lect.Notes Phys.738:1-54.

[3] Perlmutter, S., et al, 1999, Measurements of $\Omega$ and $\Lambda$ from 42 High-Redshift Supernovae. ApJ, 517, 565

[4] Riess, A.G., et al., 1998, Observational Evidence from Supernovae for an Accelerating Universe and a Cosmological Constant. The Astronomical Journal. 116, 1009 
[5] Roger Penrose, 1989, Difficulties with Inflationary Cosmology. Annals of the New York Academy of Sciences, 571,249

[6] Abhas Mitra. 2011, Why the Big Bang Model Cannot Describe the Observed Universe Having Pressure and Radiation. Journal of Modern Physics, 2,1436

[7] Steinhardt P.J., Abraham Loeb., 2014, Inflationary schism. Phys. Lett. B, 736,142

[8] P. J. E. Peebles. 2003, The Cosmological Constant and Dark Energy.Rev. Mod. Phys. 75, 559-606.

[9] P. J. E. Peebles. 2015, Dark matter.PNAS. 112 (40) 12246-12248.

[10] Hawking, S., Penrose, R., 1970, The Singularities of Gravitational Collapse and Cosmology. Proc. Roy. Soc.Lond. A, 314: 529-548

[11] Roger Penrose, 1965 gravitational collapse and space-time singularities. Phys. Rev. Lett., 14,57

[12] Hawking, S.W., 1975, Particle creation by black holes S. W. Hawking. Communications in Mathematical Physics, 43, 199, 314, 529

[13] Hubble E. P., 1929, A relation between distance and radial velocity among extra-galactic nebulae. PNAS, 15,168

[14] David W Hogg, 2000, Distance Measures in Cosmology. preprint (arXiv:9905116)

[15] Melia F., Shevchuk A. S. H., 2012, The Rh=ct universe. MNRAS, 419, 2579

[16] Moncy V. John., 2019, Rh = ct and the eternal coasting cosmological model. MNRS, 484, L35

[17] Nielsen, J.T., Guffanti, A, Sarkar S., 2016, Marginal Evidence for Cosmic Acceleration from Type Ia Supernovae. Scientific Reports, 6, 35596

[18] Seshavatharam UVS, Tatum E.T., Lakshminarayana S., 2015, On the role of gravitational self energy density in spherical flat space quantum cosmology. Journal of Applied Physical Science International, 4(4), 228.

[19] Seshavatharam UVS., Lakshminarayana S,, 2015, Toy model of Evolving Spherical Cosmology with Flatness, Angular Velocity, Temperature and Redshift. Frontiers of Astronomy, Astrophysics and Cosmology, 1,2, 90

[20] Seshavatharam UVS., Lakshminarayana S., 2020, Light speed expansion and rotation of a primordial cosmic black hole universe having internal acceleration. International Astronomy and Astrophysics Research Journal, 2,2, 9

[21] Seshavatharam UVS., Lakshminarayana S., 2020, To Correlate Galactic Dark and Visible Masses and to Fit Flat Rotation Speeds Via MOND Approach and Cosmic Angular Acceleration. International Astronomy and Astrophysics Research Journal, 2,3, 28

[22] Seshavatharam UVS., Lakshminarayana S., 2020, Light Speed Expansion and Rotation of A Very Dark Machian Universe Having Internal Acceleration. International Journal of Astronomy and Astrophysics, 10, 247

[23] Tatum E.T., Seshavatharam UVS., Lakshminarayana S., 2015, Flat Space Cosmology as a Mathematical Model of Quantum Gravity or Quantum Cosmology. International Journal of Astronomy and Astrophysics, 5, 133

[24] Tatum E.T., Seshavatharam UVS., Lakshminarayana S., 2015, The basics of flat space cosmology. International Journal of Astronomy and Astrophysics, 5,116

[25] Tatum E.T., Seshavatharam UVS., 2018, How a Realistic Linear $\mathrm{R}_{\mathrm{h}}=\mathrm{ct}$ Model of Cosmology Could Present the Illusion of Late Cosmic Acceleration. Journal of Modern Physics, 9,1397

[26] Seshavatharam UVS., Eugene Terry Tatum and Lakshminarayana S. 2021 The Large Scale Universe as a Quasi Quantum White Hole. International Astronomy and Astrophysics Research Journal 3(1): 22-42

[27] Planck 2018 results. 2020, VI. Cosmological parameters. A\&A 641, A6

[28] Di Valentino, E., Melchiorri, A. \& Silk, J. 2020, Planck evidence for a closed Universe and a possible crisis for cosmology. Nat Astron 4, 196-203.

[29] George Ellis, Julien Larena, 2020, The case for a closed universe, Astronomy \& Geophysics, 61(1), 1.38-1.40. 
[30] Will Handley, 2021, Curvature tension: evidence for a closed universe. Phys. Rev. D 103, 041301

[31] Planck Collaboration: Planck 2015 Results. XIII. Cosmological Parameters.

[32] Pengfei Li, et al., 2020, A Comprehensive Catalog of Dark Matter Halo Models for SPARC Galaxies. ApJS, 247,31

[33] Pieter van Dokkum et al., 2019, A second galaxy missing dark matter in the NGC1052 group. ApJ Let., 874, L5

[34] Shany Danieli et al., 2019, Still Missing Dark Matter: KCWI High-resolution Stellar Kinematics of NGC1052-DF2. ApJ Let., 874, L12

[35] Stacy McGaugh., 2020, Predictions and Outcomes for the Dynamics of Rotating Galaxies. Galaxies, 8,2, 35

[36] Navarro, Julio F., Frenk, Carlos S., White Simon D. M., 1996, The Structure of Cold Dark Matter Halos. The Astrophysical Journal. ApJ. 462, 563

[37] Oman Kyle et al., 2015. The unexpected diversity of dwarf galaxy rotation curves. MNRS, 452 (4), 3650

[38] Milgrom M., 1983, A modification of the Newtonian dynamics as a possible alternative to the hidden mass hypothesis. ApJ, Part 1, 270, 365

[39] Brownstein J. R. and Moffat J. W. 2006. Galaxy Rotation Curves Without NonBaryonic Dark Matter. ApJ, 636,721

[40] Federico Lelli, Stacy S. McGaugh,, James M. Schombert., 2016, SPARC: Mass Models for 175 Disk Galaxies with Spitzer Photometry and Accurate Rotation Curves. ApJ, 152,6

[41] Ogle, P.M., et al. 2019, A Break in Spiral Galaxy Scaling Relations at the Upper Limit of Galaxy Mass. The Astrophysical Journal Letters, 884, L11.

[42] Jun-Jie Wei and Fulvio Melia. 2020, Cosmology-independent Estimate of the Hubble Constant and Spatial Curvature using Time-delay Lenses and Quasars. The Astrophysical Journal, 897:127 (10pp)

[43] Godel, K., 1950, Rotating Universes in General Relativity Theory. Proceedings of the International Congress of Mathematicians in Cambridge, 1, 175-181.

[44] S.W. Hawking. 1969, On the Rotation of the universe. Mon.Not.Roy.Astron.Soc. 142, 29-141.

[45] Birch P., 1982, Is the Universe rotating? Nature, 298, 451.

[46] Rainer W. Kuhne, 1997, On the Cosmic Rotation Axis.Mod.Phys.Lett.A12:2473-2474.

[47] Li-Xin Li.1998, Effect of the Global Rotation of the Universe on the Formation of Galaxies. Gen.Rel.Grav. 30, 497-507.

[48] Su, S.-C \& Chu, M.-C. 2009, Is the Universe Rotating?. The Astrophysical Journal. 703. 354-361.

[49] Seshavatharam UVS. 2010, Physics of Rotating and Expanding Black Hole Universe. 2,7-17.

[50] Godlowski W., 2011, Global and Local Effects of Rotation: Observational Aspects. IJMPD, 20,1643

[51] Michael J. Longo, 2011, Detection of a dipole in the handedness of spiral galaxies with redshifts z 0.04. Phy. Let. B, 699,4,224.

[52] Chechin L.M, 2017, Does the Cosmological Principle Exist in the Rotating Universe? Gravitation and Cosmology, 23, 305.

[53] Shamir L, 2020, Patterns of galaxy spin directions in SDSS and Pan-STARRS show parity violation and multipoles. Astrophys Space Sci, 365,136

[54] Vladimir A Korotky, Eduard Masár Yuri N Obukhov., 2020 In the Quest for Cosmic Rotation. Universe, 6:14

[55] Michael J. Longo. Are limits on cosmic rotation from analyses of the cosmic microwave background credible? https://doi.org/10.20944/preprints202011.0520.v1.

[56] Seshavatharam UVS., Lakshminarayana S, 2020, An Integrated Model of a Light Speed Rotating Universe. International Astronomy and Astrophysics Research Journal 2(4): 7482. 
[57] Seshavatharam UVS., Lakshminarayana S, 2021, Quantum model of naturally rotating Machian cosmology and galactic applications of current cosmic angular velocity. International Journal of Advanced Astronomy, 9 (1), 18-23

[58] Mordehai Milgrom, 2020, Fast-rotating galaxies do not depart from the MOND massasymptotic-speed relation. https://arxiv.org/abs/2002.10204v1

[59] Heykhi, A., 2020, New explanation for accelerated expansion and flat galactic rotation curves. Eur. Phys. J. C 80, 25, (8 pages)

[60] ábor Rácz, László Dobos, Róbert Beck, István Szapudi and István Csabai, 2017, Concordance cosmology without dark energy, Monthly Notices of the Royal Astronomical Society: Letters, 469(1), L1-L5.

[61] Burghardt, R. 2020, Melia's Rh = ct Model Is by No Means Flat. Journal of Modern Physics, 11, 703-711.

[62] Burghardt, R. 2019, Local and Global Flatness in Cosmology. Journal of Modern Physics, 10, 1439-1453.

[63] Melia F, 2013, The R_h=ct Universe Without Inflation. A\&A 553, id A76.

[64] Melia, F. 2017, The linear growth of structure in the Rh $=$ ct Universe. MNRAS, 446, 1191-1194.

[65] Melia F, 2019, The lapse function in Friedmann-Lemaittre-Robertson-Walker cosmologies. Ann. Phys. 411, 167997.

[66] Tatum, E.T., 2018, Why Flat Space Cosmology Is Superior to Standard Inflationary Cosmology. Journal of Modern Physics, 9, 1867-1882.

[67] Tatum, E.T, 2020, Introductory Chapter: Black Holes, The Singularity Problem, and The Universe, New Ideas Concerning Black Holes and the Universe, Eugene Tatum, IntechOpen, DOI: 10.5772/intechopen.90071.

[68] Arved Sapar, 2019, A perpetual mass-generating Planckian universe.Proceedings of the Estonian Academy of Sciences, 68, 1, 1-12. 